\title{
THEORY OF ANNIHILATION GAMES
}

\author{
BY A. S. FRAENKEL AND Y. YESHA.
}

Communicated by John L. Kelley, December 23, 1975

Throughout, $R=(V(R), E(R))$ is a finite loopless digraph with vertex set $V(R)$ and edge set $E(R) \subset V(R) \times V(R)$, which may contain cycles. Let $F(u)=$ $\{v \in V(R):(u, v) \in E(R)\}, Z=$ nonnegative integers, $G F(2)^{n}=$ the $n$-fold cartesian product of $G F(2)$.

Put any number of stones on distinct vertices of $R$. Two players play alternately. Each player at his turn moves one stone from a vertex $u$ to some $v \in$ $F(u)$. If $v$ was occupied, both stones get removed (annihilation). The player making the last move wins. If there is no last move, the game is a tie.

Such an annihilation game belongs to a large class of combinatorial games discussed in [1], [3], which are analyzable by the Generalized Sprague-Grundy Function (GSG-function) $G: V(R) \rightarrow Z \cup\{\infty\}$ [1], [2], [3] with associated counter function $c: V^{f}(R) \rightarrow Z$, where $V^{f}(R)=\{u \in V(R): G(u)<\infty\}[2]$. Here $R$ is the game-graph of the game.

Our main result is the construction of a complete strategy for the game, which is polynomial in $n=|V(R)|$.

Let $C(R)$ be the game-graph of the annihilation game on $R$, also called the contrajunctive compound of $R$. If $V(R)=\left\{u_{1}, \ldots, u_{n}\right\}$, the vertices of $V(C(R))$ (= game positions) constitute the set of all $n$-tuples $\bar{u}=\left(\alpha_{1}, \ldots, \alpha_{n}\right)$ over $G F(2)$, where $\alpha_{i}=1$ if and only if a stone is on $u_{i}$. Also $(\bar{u}, \bar{v}) \subset E(C(R))$ if and only if there is a move from $\bar{u}$ to $\bar{v}$. Thus $V(C(R))$ is identical with the linear space $G F(2)^{n}$ under the operation $\oplus, \Sigma^{\prime}$ of Nim-sum (below: Generalized Nim-sum [1], [3]).

LEMMA 1. Let

$$
C^{f}(R)=\{\bar{u} \in V(C(R)): G(\bar{u})<\infty\}, \quad C_{i}(R)=\{\bar{u} \in V(C(R)): G(\bar{u})=i<\infty\} .
$$

Then

(i) $C^{f}(R)$ is a linear subspace of $V(C(R))$.

(ii) $G$ is a homomorphism from $C^{f}(R)$ onto $G F(2)^{t}$ with kernel $C_{0}(R)$ $\left(t=O\left(\log _{2} n\right)\right)$. In fact,

$$
\mathrm{G}(\bar{u})<\infty \Rightarrow G(\bar{u} \oplus \bar{v})=G(\bar{u}) \oplus G(\bar{v}) .
$$

(iii) $\left\{C_{i}(R): 0 \leqslant i<2^{t}\right\}=C^{f}(R) / C_{0}(R)$.

AMS (MOS) subject classifications (1970). Primary 05C20, 68A10, 68A20, 90D05. 
Let $L_{i}^{k}(R)=\left\{\bar{u} \in C_{i}(R):|\bar{u}|=k\right\}, L F^{k}(R)=\left\{\bar{u} \in C^{f}(R):|\bar{u}|=k\right\}$, $\mathfrak{P}(S)=$ linear span of $S, \Im_{0}(R)=L_{0}^{4}(R) \cup L_{0}^{2}(R) \cup L_{0}^{1}(R), \Im^{f}(R)=\wp_{0}(R) \cup$ $L F^{2}(R)$.

LEMMA 2. (i) $C_{0}(R)=\Re\left(\Im_{0}(R)\right)$.

(ii) $C^{f}(R)=\mathfrak{q}\left(\Im^{f}(R)\right)$.

(iii) There exists a basis $\beta^{f}=\left(\bar{u}_{1}, \ldots, \bar{u}_{m}, \bar{v}_{1}, \ldots, \bar{v}_{t}\right)$ for $C^{f}(R)$ such that $\beta_{0}=\left(\bar{u}_{1}, \ldots, \bar{u}_{m}\right)$ is a basis of $C_{0}(R)$ and $\bar{v}_{i} \in L_{j(i)}^{2}(R)$, where $j(i)=2^{i-1}$ $(1 \leqslant i \leqslant t)$.

Note. For $m \geqslant 0$, denote by $C^{(m)}(R)$ the subgraph of $C(R)$ with vertices $\bar{u}$ satisfying $|\bar{u}| \leqslant m$. Then $C^{(m)}(R)$ has $O\left(n^{m}\right)$ vertices, and $\bar{u} \in V\left(C^{(m)}(R)\right) \Rightarrow$ $F(\bar{u}) \subset V\left(C^{(m)}(R)\right)$. Hence $G$ on $C^{(m)}(R)$ can be computed from $C^{(m)}(R)$ alone. In particular, $\subseteq^{f}(R) \subset V\left(C^{(4)}(R)\right)$. Hence $\subseteq^{f}(R)$ can be computed in $O\left(n^{6}\right)$ steps using standard algorithms for computing the GSG-function [1] .

THEOREM 1. There exists an $n \times n$ matrix $\Gamma$ over $G F(2)$ which can be computed polynomially, such that for every $\bar{u} \in V(C(R))$ we have $\Gamma \cdot \bar{u}^{\prime}=$ $\left(\epsilon_{1}, \ldots, \epsilon_{n}\right)^{\prime}$, where

$$
\bar{u}=\sum_{i=1}^{m} \epsilon_{i} \bar{u}_{i} \oplus \sum_{j=1}^{t} \epsilon_{m+j} \bar{v}_{j} \oplus \sum_{k=1}^{n-m-t} \epsilon_{m+t+k} \bar{z}_{k},
$$

and $\left(\bar{z}_{1}, \ldots, \bar{z}_{k}\right)$ is a basis of a complementary space of $C^{f}(R)$. Moreover, letting $Q(\bar{u})=\left(\epsilon_{n}, \epsilon_{n-1}, \ldots, \epsilon_{m+1}\right), Q$ is a homomorphism from $V(C(R))$ onto $G F(2)^{n-m}$ with kernel $C_{0}(R)$, such that $G(\bar{u})=\bar{Q}(\bar{u})=\Sigma_{i=1}^{\prime n} \epsilon_{m+i} 2^{i-1}$ if $\left(\epsilon_{n}, \epsilon_{n-1}, \ldots, \epsilon_{m+t+1}\right)=(0,0, \ldots, 0) ; G(\bar{u})=\infty$ otherwise.

Conclusion 1. The $N, P, T$ classification [1], [2], [3] and the GSGfunction of any $\bar{u}=\left(\alpha_{1}, \ldots, \alpha_{n}\right)$ can be computed polynomially. In particular, the values $\bar{Q}\left(\bar{u}_{i}\right)$, where $\bar{u}_{i}=\left(\epsilon_{1}, \ldots, \epsilon_{n}\right), \epsilon_{i}=1, \epsilon_{j}=0(j \neq i ; i=1, \ldots, n)$, determine $G(\bar{u})$. Indeed,

$$
Q(\bar{u})=\sum_{\alpha_{i}=1}^{\prime} Q\left(\bar{u}_{i}\right)=\left(\delta_{n}, \delta_{n-1}, \ldots, \delta_{m+1}\right),
$$

and so $G(\bar{u})=Q(\bar{u})$ if $\delta_{n}=\cdots=\delta_{m+t+1}=0 ; G(\bar{u})=\infty$ otherwise. This, however, does not yet guarantee the realization of a winning strategy, because of possible cycling.

Let $\bar{u} \in P=C_{0}(R)$. Then $\bar{u}$ has a representation $\tilde{u}=\left(\bar{y}_{1}, \ldots, \bar{y}_{k}\right) \subset$ $\subseteq_{0}(R)(k \leqslant n)$ in the sense that $\bar{u}=\Sigma_{i=1}^{\prime k} \bar{y}_{i}$. For example, initially we may have $\tilde{u} \subset \beta_{0}$. Let $c$ be a monotonic counter function on $C^{(4)}(R)$ (i.e., $G(\bar{u})<$ $G(\bar{v}) \Rightarrow c(\bar{u})<c(\bar{v}))$. We can choose $c(\bar{u})=O\left(n^{4}\right)$ for all $\bar{u} \in V\left(C^{(4)}(R)\right)$. Let $\widetilde{c}(\tilde{u})=\Sigma_{i=1}^{k} c\left(\bar{y}_{i}\right)$. Then $\widetilde{c}=O\left(n^{5}\right)$.

THEOREM 2. There is a function $\Lambda_{0}$ which can be computed polynomially, such that for every $\bar{u} \in C_{0}(R)$ and every $\bar{v} \in F(\bar{u})$, 


$$
\begin{gathered}
\Lambda_{0}(\tilde{u}, \bar{v})=\widetilde{w}=\left(\bar{w}_{1}, \ldots, \bar{w}_{k}\right) \subset \Theta_{0}(R), \\
\bar{w}=\sum_{i=1}^{k}{ }^{\prime} \bar{w}_{i} \in F(\bar{v}) \cap P, \\
\tilde{c}(\tilde{w})<\tilde{c}(\tilde{u}) .
\end{gathered}
$$

Note. The representation $\widetilde{w}$ is obtained from $\tilde{u}$ in a bounded number of transformations. Details are omitted.

ConCLUSION 2. Using $\Lambda_{0}$ and starting from any $N$-position, every annihilation game can be won in $O\left(n^{5}\right)$ moves using polynomial computation time throughout. The function $\Lambda_{0}$ implies a winning strategy in the wide sense [3]. A bounded number of cycles may be traversed in realizing the strategy (but no cycling takes place in the "representation space"). We do not know if a winning strategy in the narrow sense exists which is always polynomial.

Further results, ramifications and proofs will appear elsewhere.

\section{REFERENCES}

1. A. S. Fraenkel and Y. Perl, Constructions in combinatorial games with cycles, Colloq. Math. Soc. János Bolyai, no. 10, Proc. Internat. Colloq. on Infinite and Finite Sets (Keszthely, Hungary, 1973; A. Hajnal, R. Rado and V. D. Sós, editors), Vol. 2, North-Holland, Amsterdam, 1975, pp. 667-699.

2. A. S. Fraenkel and U. Tassa, Strategy for a class of games with dynamic ties, Comput. Math. Appl. 1 (1975), 237-254.

3. C. A. B. Smith, Graphs and composite games, J. Combinatorial Theory 1 (1966), 51-81. MR $33 \# 2572$.

DEPARTMENT OF APPLIED MATHEMATICS, THE WEIZMANN INSTITUTE OF SCIENCE, REHOVOT, ISRAEL 